\title{
Chromatin and higher-order chromosome organization shape the recombination landscape in C. elegans
}

\author{
Chitra V Kotwaliwale ${ }^{1,2^{*}}$, Sasha A Langley ${ }^{3}$, Andrea C Dose ${ }^{1}$, Clara W Wang ${ }^{2}$, Abby F Dernburg ${ }^{1,2}$ \\ From Epigenetics and Chromatin: Interactions and processes \\ Boston, MA, USA. 11-13 March 2013
}

Meiotic recombination generates crossovers, which physically link homologous chromosomes, and direct their segregation to opposite poles. Despite the benefits, crossovers are restricted both in number as well as spatially. Crossovers in the nematode C. elegans occur most frequently within the distal regions (or "arms") of the chromosomes, which comprise $\sim 50 \%$ of the genome, but $\sim 90 \%$ of the genetic map length. Meiotic recombination initiates with the formation of programmed double strand breaks (DSBs) catalyzed by the SPO-11 enzyme. It has been unclear whether the crossover bias in C. elegans is a consequence of DSB distribution, or instead reflects a mechanism that biases the downstream repair outcome of DSBs. In order to address this, we generated a genome-wide map of meiotic DSBs by mapping the distribution of the sole C. elegans RecA-like recombinase, RAD-51, by ChIP-seq. We have found that the RAD-51 binding pattern is strikingly similar to the known recombination pattern in C. elegans. The majority of RAD-51 binding sites occur on chromosome arms. Comparing RAD-51 distribution relative to functional genomic elements revealed that RAD-51 is particularly enriched in intronic DNA while coding sequences are underrepresented in RAD-51 occupied regions. We find that monomethylation of histone $\mathrm{H} 3$ lysine 36 (H3K36me1), which has not previously been associated with meiotic recombination, is highly correlated with break site preference. H3K36me1 is particularly enriched over active genes with high intron content, suggesting that the specific chromatin environment associated with these genes

'Department of Molecular and Cell Biology, University of California, Berkeley, CA 94720, USA

Full list of author information is available at the end of the article favors DSB formation in C. elegans. We have also identified a sequence motif that is highly overrepresented in DSB sites. Although the DSB pattern is highly correlated with crossover frequency, there are interesting exceptions. In particular, the sub-telomeric regions are completely devoid of crossovers but are active DSB sites. We have also identified a DNA sequence motif that appears to be enriched within DSB sites suggesting that sequence specific factors may have a role in DSB specification in C. elegans.

In a parallel study, we have developed an Illumina Golden Gate SNP array to rapidly measure genomewide recombination rates in C. elegans. Using this array we measured meiotic recombination rates in met-2 (n4256) mutant animals. MET-2 is one of the two histone methyltransferases responsible for H3K9 methylation, a histone mark associated with heterochromatin. We find that the genetic map is significantly altered in met-2(n4256) mutant animals. In particular, regions that are normally enriched for H3K9 methylation show elevated recombination rates in met-2(n4256) mutants. We are currently investigating whether this change in the genetic map is the result of altered DSB distribution. Taken together, our data provide new insights into the role of chromatin in meiotic recombination. Because meiotic recombination generates genetic diversity, our data implicates chromatin as a key player in the evolution of genomes.

\footnotetext{
Author details

${ }^{1}$ Department of Molecular and Cell Biology, University of California, Berkeley, CA 94720, USA. ${ }^{2}$ Howard Hughes Medical Institute, Chevy Chase, MD 20815, USA. ${ }^{3}$ Department of Genome Dynamics, Life Sciences Division, Lawrence Berkeley National Laboratory, Berkeley, CA 94720, USA.
} 
doi:10.1186/1756-8935-6-S1-017

Cite this article as: Kotwaliwale et al:: Chromatin and higher-order chromosome organization shape the recombination landscape in C. elegans. Epigenetics \& Chromatin 2013 6(Suppl 1):017.

Submit your next manuscript to BioMed Central and take full advantage of:

- Convenient online submission

- Thorough peer review

- No space constraints or color figure charges

- Immediate publication on acceptance

- Inclusion in PubMed, CAS, Scopus and Google Scholar

- Research which is freely available for redistribution

Submit your manuscript at www.biomedcentral.com/submit 